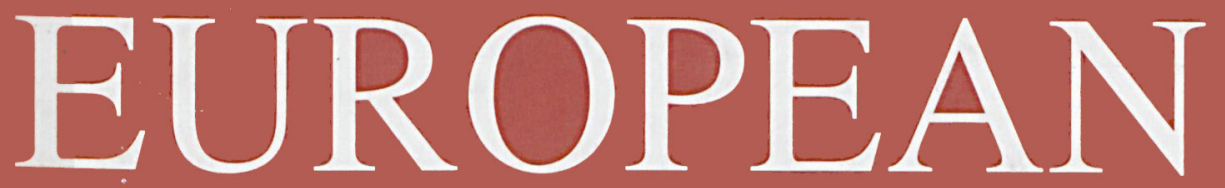

VOL 11/No 3

\title{
1996
}

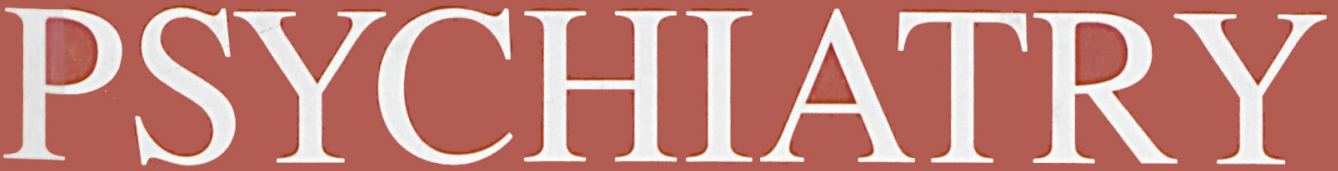

ISSN 0924-9338

SCSPED II (3) 109-164 PSYPEK (11) (3)

\section{Issues of concern in the standardization and harmonization of psychotropic drug trials in Europe}

THE JOURNAL OF THE ASSOCIATION OF EUROPEAN PSYCHIATRISTS

ECST STRASBOURG, 26-27 May 1994

Methodological approaches to the long-term study of antidepressants F Rouillon, LSteru, S Wood (Colombes, Le Kremlin-Bicêtre, France)

Long-term studies in depression - relapse versus remission. Long-term studies with antidepressants - methodological aspects for consideration

$\checkmark$ Pedersen (Copenhagen-Valby, Denmark)

Long-term studies in depression - relapse, recurrence, and reliability of outcome measures: discussion

JMA Sitsen (Oss, The Netherlands)

Quality of hife measurements in major depression

$P$ Bech (Hiller $\phi$ d, Denmark)

Long-term studies of antipsychotic drugs in schizophrenia

T Lewander (Södertälje, Uppsala, Sweden)

Long-term studies of antipsychotic drugs in schizophrenia - discussion

HJ Möller (Munich, Germany)......

Cost-effectiveness in the treatment of schizophrenia

MRJ Knapp (London, UK)

Key considerations for long-term studies with anxiolytics

M Placchi (Braine-l'Alleud, Belgium)

Designing dementia treatment studies: diagnosis, efficacy criteria, and duration

$R$ Spiegel, P Irwin (Basel, Switzerland; East Hanover, NJ, USA)

Measuring patient benefit in mental illness

C Gudex (York, UK)

Cost-utility in CNS drug trials

F Ziegler (Broendby, Denmark)

Instructions to authors

inside back cover

Cited/abstracted in: Biological Abstracts/Biosis, CNRS/Pascal, Current Contents/Clinical Medicine and Social and Behavioral Sciences, Excerpta Medica/EMbase, Psychological Abstracts

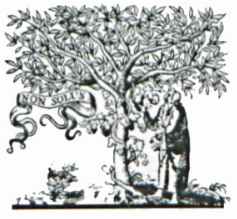




\section{EUROPEAN PSYCHIATRY}

\section{THE JOURNAL OF THE ASSOCIATION OF EUROPEAN PSYCHIATRISTS}

Editors: C Ballus (Barcelona), JD Guelfi (Paris/Villejuif), H Heimann (Tübingen), R Murray (London)

Co-Editors: M Maj (Naples), CB Pull (Luxembourg), M Ackenheil (Munich), P Bech (Copenhagen)

Deputy editors: P Boyer, Y Lecrubier (Paris). Secretariat: Hôpital de la Salpêtrière, Pavillon Clérambault, 47, bd de l'Hôpital, 75651 Paris Cedex 13, France. Tel: 33 (1) 42161656 . Fax: 33 (1) 45852800

Advisory board

J Adès, Colombes, France

HS Akiskal, Rockville, MD, USA

NC Andreasen, Iowa City, IA, USA

J Angst, Zurich, Switzerland

M Ansseau, Liège, Belgium

P Baumann, Lausanne, Switzerland

H Beckmann, Würzburg, Germany

P Berner, Vienna, Austria

A Bertelsen, Risskov, Denmark

J Bibert, Cadiz, Spain

$\mathrm{J}$ Birley, London, UK

JC Bisserbe, Meudon, France

B Bondy, Munich, Germany

JP Boulenger, Sherbrooke, Canada

M Bourgeois, Bordeaux, France

F Brambilla, Milan, Italy

I Brockington, Birmingham, UK

A Clare, Dublin, Ireland

F Clerget-Darpoux, Paris, France

V Conde Lopez, Valladolid, Spain

S Consoli, Paris, France

P Cosyns, Antwerp, Belgium

J Cottraux, Lyon, France

$M$ von Cranach, Kaufbeuren, Germany

A Dahl, Oslo, Norway

JM Danion, Strasbourg, France

JFW Deakin, Manchester, UK

$\mathrm{M}$ de Bonis, Le Kremlin Bicêtre, Paris, France

M Fichter, Prien am Chiemsee, Germany

H Freeman, London, UK

HJ Gaertner, Tübingen, Germany

D Goldberg, Manchester, $U K$

I Hand, Hamburg, Germany

H Häfner, Mannheim, Germany

T Helgason, Reykjavik, Iceland

H Hippius, Munich, Germany

A Jablenski, Sofia, Bulgaria

E Johnston, Edinburgh, UK

S Kasper, Vienna, Austria

M Kastrup, Hvidovre, Denmark

D Kemali, Naples, Italy

R Kendell, Edinburgh, UK

D Klein, New York, NY, USA

R Klein, New York, NY, USA

S Langer, Paris, France

J Lellouch, Villejuif, France

P Lemoine, Lyon, Paris

T Lemperière, Colombes, France

JP Lépine, Paris, France

OM Lesch, Vienna, Austria

SW Lewis, London, UK

H Lôo, Paris, France

JJ Lopez-Ibor, Madrid, Spain

P McGuffin, Cardiff, UK

W Maier, Mainz, Germany

A Mann, London, UK

K Mann, Tübingen, Germany

I Marks, London, UK

J Marlet, Venray; The Netherlands

J Massanna, Barcelona, Spain

J Mendlewicz, Brussels, Belgium

HJ Möller, Munich, Germany

N Müller, Munich, Germany
M Musalek, Vienna, Austria

D Naber, Munich, Germany

E O'Callaghan, Dublin, Ireland

Y Ono, Tokyo, Japan

M Patris, Strasbourg, France

J Pellet, Saint-Etienne, France

C Perris, Umeä, Sweden

P Pichot, Paris, France

T Pohlmächer, Munich, Germany.

H Pope, Belmont, MA, USA

AJ Puech, Paris, France

G Racagni, Milan, Italy.

N Retterstøl, Oslo, Norway

M A Ron, London, UK

R Rosenberg, Risskov, Denmark

M Roth, Cambridge, UK

F Rouillon, Colombes, France

J Saiz-Ruiz, Madrid, Spain

A Sanchez-Blanque, Zaragoza, Spain

N Sartorius, Geneva, Switzerland

F Schulsinger, Copenhagen, Denmark

G Sedvall, Stockholm, Sweden

L Singer, Strasbourg, France

CN Stefanis, Athens, Greece

E Straube, Tübingen, Germany

E Taylor, London, $U K$

P Taylor, London, UK

L Träskman-Bendz, Lund, Sweden

J Vallejo, Barcelona, Spain

L Waintraub, Paris, France

D Widlöcher, Paris, France

J Wilmotte, Charleroi, Belgium

J Wing, London, UK

FT Zimmer, Tübingen, Germany

J Zohar, Beer-Sheva, Israel

F Ferrero, Geneva, Switzerland

Association of European Psychiatrists

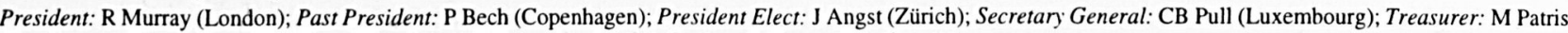
(Strasbourg); Counsellors: H Höfner (Mannheim), Y Lecrubier (Paris); Section: L Singer (Strasbourg)

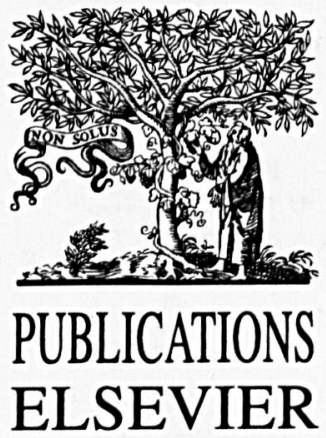

A division of EDITIONS SCIENTIFIQUES ET MÉDICALES ELSEVIER SAS

A member of Elsevier Science

141, rue de Javel

75747 Paris cedex 15, France
MEDICAL DEPARTMENT

Tel: 33 (1) 45589026 - Fax: 33 (1) 45589422

Desk editor - Valerie-Ann Libert: 33 (1) 45589031

Advertising - Anne Blanvillain: 33 (1) 45589024

Consulting translator: P Rousseau-Cunningham

\section{SUBSCRIPTIONS - Tel: 33 (1) 45589067 - Fax: 33 (1) 45589424}

1996 (Vol 11) Annual subscription; 8 issues:

- France: 1885 FF • European Union (with VAT registration no) and rest of the world: 2115 FF $\bullet$ European Union (individuals): $2159 \mathrm{FF} \bullet$ North, Central and South America: 413 US $\$ \bullet$ Students (copy of the student card requested) France: $1131 \mathrm{FF}$; rest of the world: $1296 \mathrm{FF}$.

Including air delivery.

Address order and payment to: Éditions scientifiques et médicales Elsevier

- by check or credit card (CB, MasterCard or Visa; indicate no and expiration date)

- by transfer: CCP Paris no 30041000011904540 H 020/70.

- Subscriptions begin 4 weeks after receipt of payment and start with the first issue of the calendar year

- Back issues and volumes are available from the publisher.

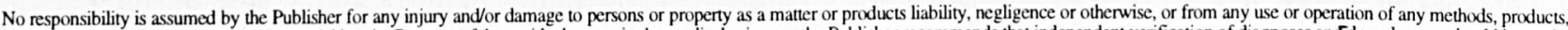

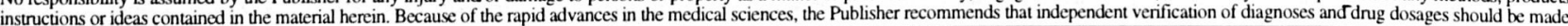

(c) 1996 Éditions scientifiques et médicales Elsevier, Pari

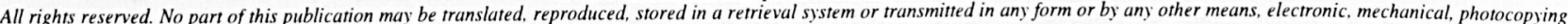
recording or otherwise, without prior permission of the publisher. 